\title{
Dermoscopy, Optical Coherence Tomography, and Histological Correlation of Pseudoxanthoma Elasticum
}

\author{
Flavia Persechino ${ }^{1,2}$, Domenico Giordano ${ }^{3}$, Chiara D. Marini ${ }^{4}$, Chiara Franceschini ${ }^{2}$, \\ Marco Ardigò ${ }^{2}$, Severino Persechino ${ }^{3}$
}

\begin{abstract}
1 Department of Clinical and Molecular Medicine, Sapienza University of Rome, Italy
2 Clinical Dermatology, San Gallicano Dermatological Institute, IRCCS, Rome, Italy

3 NESMOS Department, Dermatology Unit, Sant'Andrea Hospital Faculty of Medicine \& Department of Psychology, Sapienza University of Rome, Italy

4 Surgical Pathology Units, Department of Clinical and Molecular Medicine, Ospedale Sant'Andrea, Sapienza University of Rome, Italy
\end{abstract}

Key words: dermoscopy, pseudoxanthoma elasticum, optical coherence tomography, histology, elastic fibers

Citation: Persechino F, Giordano D, Marini CD, Franceschini C, Ardigò M, Persechino S. Dermoscopy, optical coherence tomography, and histological correlation of pseudoxanthoma elasticum. Dermatol Pract Concept. 2019;9(3):209-210. DOI: https://doi.org/10.5826/ dpc.0903a07

Accepted: April 25, 2019; Published: July 31, 2019

Copyright: @2019 Persechino et al. This is an open-access article distributed under the terms of the Creative Commons Attribution License, which permits unrestricted use, distribution, and reproduction in any medium, provided the original author and source are credited.

Funding: None.

Competing interests: The authors have no conflicts of interest to disclose.

Authorship: All authors have contributed significantly to this publication.

Corresponding author: Severino Persechino, MD, NESMOS Department, Dermatology Unit, Sant'Andrea Hospital Faculty of Medicine \& Department of Psychology, Sapienza University of Rome, Via di Grottarossa, 1035, 00189 Rome, Italy. Email: severino.persechino@ uniroma1.it

\section{Case Presentation}

We propose the case of a 17 -year-old female patient with multiple, soft, yellowish papules symmetrically distributed on the neck (Figure 1A). Dermoscopy showed a yellowish orange area associated with reddish and whitish areas [1] (Figure 1B). Optical coherence tomography (OCT) revealed normal epidermis associated with clusters of horizontally oriented dermal aggregates with peripheral clefts (Figure 1C). A biopsy was performed in the same site of previous noninvasive imaging techniques. Histology disclosed the presence of altered elastic fibers in the upper and reticular dermis with extension to the papillary dermis stained with hematoxylin and eosin (Figure 1D). Pseudoxanthoma elasticum (PXE) is a rare disorder concerning elastolytic fibers due to a genetic mutation, with systemic alterations as mainly cardiac and ophthalmological complications due to abnormalities of elastic fibers.

\section{Teaching Point}

We believe that this is the first report of PXE with dermoscopy correlated to OCT and histology. OCT may be useful for PXE confirmation showing specific dermal features.

\section{Reference}

1. Nasca MR, Lacarubba F, Caltabiano R, Verzi AE, Micali G. Perforating pseudoxanthoma elasticum with secondary elastosis perforans serpiginosa-like changes: dermoscopy, confocal microscopy and histopathological correlation. J Cutan Pathol. 2016;43(11):1021-1024. 

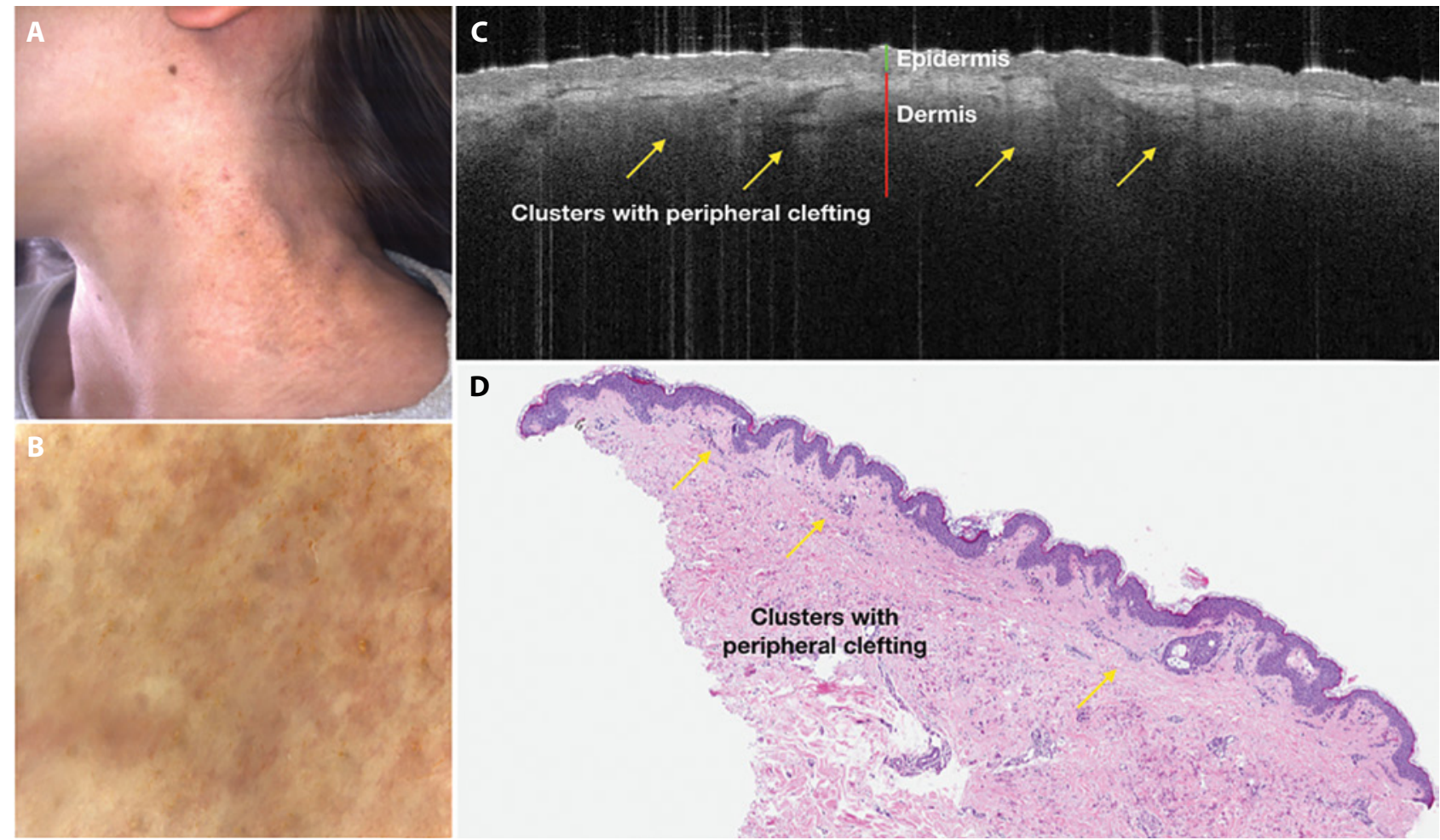

D

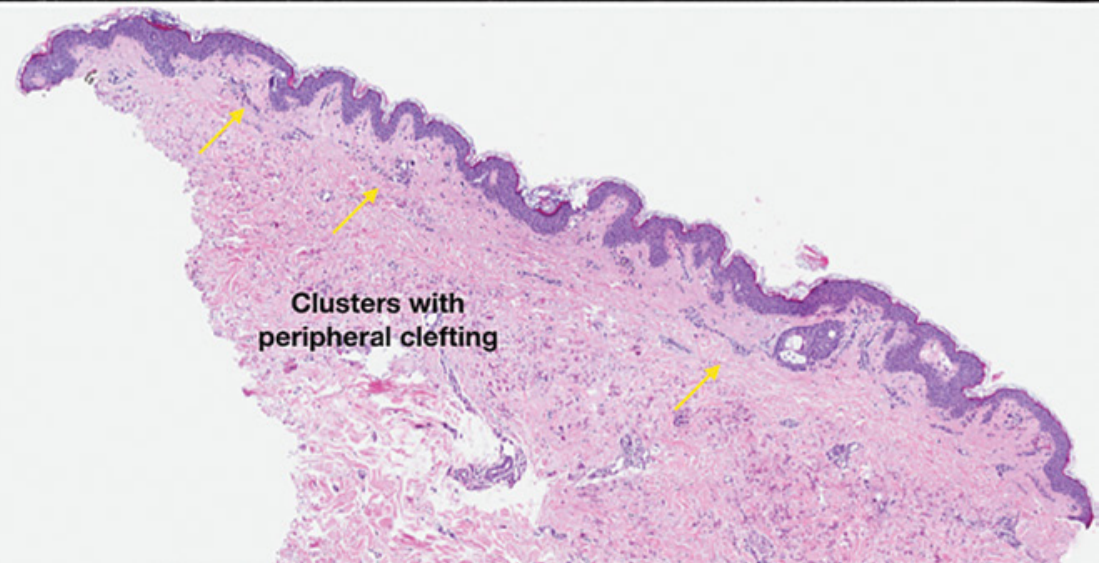

Figure 1. (A) Clinical photograph of PXE. (B) Dermoscopy of PXE. (C) OCT revealed normal epidermis associated with clusters of horizontally oriented dermal aggregates with peripheral clefts. (D) Histology disclosed the presence of altered elastic fibers in the upper and reticular dermis with extension to the papillary dermis stained with hematoxylin and eosin. [Copyright: @2019 Persechino et al.] 